\title{
The effect of 19-gauge endobronchial ultrasound-guided transbronchial needle aspiration biopsies on characterisation of malignant and benign disease. The Bristol experience
}

\author{
Rachel C. Jones', Nidhi Bhatt², Andrew R.L. Medford1 \\ 1 North Bristol Lung Centre, Southmead Hospital, North Bristol NHS Trust, Westbury-on-Trym, Bristol \\ 2 Department of Cellular Pathology, Pathology Sciences Building, Southmead Hospital, North Bristol NHS \\ Trust, Westbury-on-Trym, Bristol, UK
}

\begin{abstract}
EBUS-TBNA is a recent mediastinal staging and diagnostic technique. We have previously reported superior characterisation with $21 \mathrm{G}$ biopsies over $22 \mathrm{G}$ biopsies for benign and malignant mediastinal nodes. A new 19G needle now exists but there are limited studies. We hypothesised $19 \mathrm{G}$ biopsies would improve both benign and malignant characterisation due to larger samples. We retrospectively analysed sequential patients referred for EBUS-TBNA with unexplained mediastinal adenopathy performed with 19G, $21 \mathrm{G}$ and $22 \mathrm{G}$ needles respectively (100 patients each). Contingency table analysis was performed. There were no complications. Sensitivity for malignancy was highest in the $19 \mathrm{G}$ group ( $95.7 \%$ versus $94.7 \%$ and $87.5 \%$, respectively). The $19 \mathrm{G}$ group had higher mean lymph node size $(19.4 \mathrm{~mm}$ versus $18.6 \mathrm{~mm}$ and $13.5 \mathrm{~mm}$, respectively), the highest proportion of lymphoma ( $9 \%$ versus
\end{abstract}

Corresponding author: Dr Andrew RL Medford, Consultant \& Honorary Senior Lecturer in Respiratory Medicine, North Bristol Lung Centre \& University of Bristol, Southmead Hospital, Westbury-on-Trym, Bristol BS10 5NB, UK.

Tel. +44.1174146340 - Fax +44.1174149496 .

E-mail: andrewmedford@hotmail.com

Key words: EBUS-TBNA; 19-gauge; characterisation; benign mediastinal disease; cancer.

Contributions: ARLM, RCJ, study concept and design, data collection, analysis and interpretation, paper drafting; NB, provided further data and contribution to study design. All authors revised the manuscript critically agree to be accountable for all aspects of work.

Conflict of interest: The authors have no conflict of interest or funding to declare.

Received for publication: 21 February 2018

Accepted for publication: 3 June 2018

(C) Copyright R.C. Jones et al., 2018

Tipografia PI-ME Editrice, Italy

Monaldi Archives for Chest Disease 2018; 88:915

doi: 10.4081/monaldi.2018.915

This article is distributed under the terms of the Creative Commons Attribution Noncommercial License (by-nc 4.0) which permits any noncommercial use, distribution, and reproduction in any medium, provided the original author(s) and source are credited.
$5 \%$ and $0 \%$, respectively), the lowest proportion of NSCLC-NOS ( $2 \%$ versus $12 \%$ and $5 \%$, respectively), the highest proportion of subcharacterised benign disease $(89.6 \%$ versus $69.8 \%$ and $37.9 \%$, respectively). This large single centre retrospective UK study suggests the 19G needle appears safe with the suggestion of better sensitivity for malignancy subcharacterisation of benign disease but this requires further study in adequately powered comparative controlled studies with univariate and multivariate analysis.

\section{Introduction}

Endobronchial ultrasound-guided transbronchial needle aspiration (EBUS-TBNA) is a commonly used minimally invasive mediastinal node sampling technique used for lung cancer staging and diagnosis, but also diagnosis of unexplained other malignant and benign mediastinal pathologies or drainage of mediastinal cysts [1]. We have previous reported superiority of the $21 \mathrm{G}$ needle over $22 \mathrm{G}$ needle in characterising benign mediastinal lesions and subcharacterising nonsmall cell lung cancer (NSCLC) in a retrospective observational study although data from other studies show conflicting results likely due to different methodologies of specimen processing and study design [2,3]. A more recent 19G EBUS-TBNA needle [4-6] has been developed with the potential benefits of better flexibility and larger needle size to improve diagnosis. We hypothesised the $19 \mathrm{G}$ biopsies would be superior to $21 \mathrm{G}$ and $22 \mathrm{G}$ in subcharacterisation of NSCLC and benign disease.

\section{Patients and Methods}

Having consulted our local research and ethics committee, no specific ethical approval was required as this study was part of the ongoing standard of care for patients undergoing EBUS-TBNA. All results were reviewed at multidisciplinary team meetings. They were reported by a lung pathologist (and double-reported with another lung pathologist) in order to direct further clinical management.

We carried out a retrospective analysis on the preceding 300 patients (100 consecutive patients with 19G, 21G and 22G EBUS-TBNA needle biopsies, respectively) referred to a university teaching hospital tertiary EBUS centre in South West England between October 2014 and April 2017. Our EBUS service receives extra-regional referrals as well as from centres within the Avon, Somerset and Wiltshire Cancer Network as well as the local population.

EBUS-TBNA was performed under local anaesthesia with conscious sedation [7] (midazolam and fentanyl) using a dedicated convex probe ultrasound bronchoscope (Olympus BF-UC260FW, Olympus, Tokyo, 
Japan) as previous described [8]. All EBUS procedures were performed by three trained operators.

Following direct visualization of the tracheobronchial tree, the mediastinal nodes or lesions were localized under ultrasound. Dedicated 19G, $21 \mathrm{G}$ or 22G EBUS-TBNA needles (Olympus ViziShot, NA-U402SX4019, NA-201SX-4021 and NA-201SX-4022) were used for sampling at the discretion of the operator. Briefly, usual practice was to use $19 \mathrm{G}$ or $21 \mathrm{G}$ needles for suspected benign disease or lymphoma, or larger unexplained nodes; and use 22G needles for cancer staging or diagnosis or smaller unexplained nodes. The number of stations sampled was determined by the operator except that a staging EBUS would involve sampling of at least 3 stations and in general 2 passes were carried out per station sampling N3 nodes first then N2 nodes. Only one size of sampling needle was used on each patient.

EBUS-TBNA specimens were transferred from the microcassettes in which they were placed in at the time of the procedure, into a tissue cassette as previously reported [9]. They were then processed as histology specimens, fixed in formalin, paraffin-embedded and stained with H\&E (NB: our centre does not have a rapid on-site evaluation for cytology (ROSE) service [10]). Lung pathologists were blinded to needle gauge and double-reported all samples.

Specimens were only classified as non-small cell lung cancer, not otherwise specified (NSCLC-NOS) when immunohistochemistry had been performed and was inconclusive, or where there was insufficient material for immunohistochemistry precluding further subtyping in an undifferentiated malignant EBUS-TBNA specimen. Samples were considered to be adequate if lymphocytes were identified at cytological examination or if a definitive diagnosis was achieved.

Contingency table statistical analysis using Fisher's exact test was performed (GraphPad Prism version 7, GraphPad Software Inc, San Diego, CA, USA) to compare sensitivity, specificity, negative predictive value and accuracy of 19G, 21G and 22G EBUS-TBNA needles, and the ability to subcharacterise NSCLC and benign lesions. ANOVA was used for comparison of normal data, Chi-Squared test for other contingency table data.
Each patient was either followed up clinico-radiologically for a minimum of 12 months to confirm that negative samples were indeed 'true negative' or underwent mediastinoscopy if clinically appropriate. If a subsequent diagnosis of malignancy was made, then these were regarded as 'false negative' EBUS-TBNA results.

'True positive' malignant EBUS-TBNA biopsy results were not verified with mediastinoscopy as this was not clinical practice in our institution.

Importantly, diagnosis of sarcoidosis required strict histological criteria as well (non-necrotizing well-formed granulomas, as identified by cytological or histological analysis, together with negative mycobacterial and fungal stains and cultures in the absence of malignancy with compatible radiology and clinical features, as well as 12 month clinical and radiological follow-up) to ensure this was true sarcoidosis and not a sarcoidal reaction to malignancy [11].

The proportion of subcharacterised benign disease (with a positive benign diagnosis via EBUS-TBNA) was calculated dividing by the total number of benign cases (uncharacterised and subcharacterised) after 12-month clinico-radiological follow-up. Nodes secondary to interstitial lung disease (other than sarcoidosis) or bronchiectasis/infection were defined following careful 12-month clinico-radiological follow-up with no other explanation for the adenopathy.

\section{Results}

Patient demographics for the 300 patients are shown in Table 1. There were no complications or overnight stay in any of the patient groups with a consistent male preponderance throughout but lower age in the $19 \mathrm{G}$ and $21 \mathrm{G}$ groups.

Characteristics of the lymph nodes sampled are shown in Table 2. The $21 \mathrm{G}$ and $19 \mathrm{G}$ groups had higher mean node size (19.4 mm versus $18.6 \mathrm{~mm}$ and $13.5 \mathrm{~mm}$, respectively) with a similar sampling distribution across the groups with commonest stations sampled being subcarinal, and bihilar stations.

Table 1. Patients demographics.

\begin{tabular}{lccc} 
& $19 G$ & $21 G$ & $22 G$ \\
Male (\%) & 59 & 57 & 54 \\
\hline Female (\%) & 41 & 43 & 46 \\
\hline Mean (SD) age (years) & $58.7(16.2)$ & $60.9(14.1)$ & $67.3(11.4)$ \\
\hline
\end{tabular}

Table 2. Characteristics of nodes.

\begin{tabular}{|c|c|c|c|c|}
\hline & $19 G$ & $21 G$ & $22 \mathrm{G}$ & p values \\
\hline Mean (SD) size of nodes (mm) & $19.4(6)$ & $18.6(7.1)$ & $13.5(4.4)$ & $<0.05$ \\
\hline Total number of nodes sampled & 195 & 188 & 183 & 0.75 \\
\hline Mean number of nodes sampled & 1.95 & 1.88 & 1.83 & \\
\hline \multicolumn{5}{|c|}{ Distribution of nodes by number (\%) } \\
\hline $2 \mathrm{R}$ & 0 & $1(1)$ & $1(1)$ & 0.92 \\
\hline $4 \mathrm{R}$ & $18(9)$ & $17(9)$ & $19(10)$ & \\
\hline $4 \mathrm{~L}$ & $13(7)$ & $9(5)$ & $13(7)$ & \\
\hline 7 & $83(43)$ & $84(45)$ & $65(35)$ & \\
\hline $10 \mathrm{R}$ & $4(2)$ & $6(3)$ & $6(3)$ & \\
\hline $10 \mathrm{~L}$ & $4(2)$ & $3(2)$ & $3(2)$ & \\
\hline $11 \mathrm{R}$ & $30(15)$ & $35(19)$ & $34(18)$ & \\
\hline $11 \mathrm{~L}$ & $38(19)$ & $27(14)$ & $37(20)$ & \\
\hline Precarinal & $4(2)$ & $3(2)$ & $6(3)$ & \\
\hline Mass & $1(1)$ & $3(2)$ & $3(2)$ & \\
\hline
\end{tabular}


Diagnostic performance for malignancy for the respective needles is shown in Table 3. All pathological diagnoses were all agreed on double reporting, and there was no inter-observer variability between the two pathologists for any of the samples. Sensitivity was highest in the 19G group, with identical higher accuracy in the 19G and 21G groups (96.0\%). Prevalence of malignancy was highest in the 19G group ( $92.0 \%$ versus $75.0 \%$ and $56.0 \%$, respectively) which may account for the lower negative predictive value $(66.7 \%$ versus $86.2 \%$ and $86.3 \%$, respectively) here and higher mean node size.

Subcharacterisation of malignancy for each group is shown in Table 4. The proportion of small cell lung cancer was lowest in 22G which might also reflect the lower mean lymph node size in this group. Adenocarcinoma and squamous cell carcinoma type NSCLC accounted for the highest proportion in all groups. The $19 \mathrm{G}$ group had the highest proportion of malignancy, the highest proportion of lymphoma cases
( $9 \%$ versus $5 \%$ and $0 \%$, respectively) and lowest proportion of NSCLCNOS cases ( $2 \%$ versus $12 \%$ and $5 \%$, respectively). Each group contained a similar proportion of metastatic non-lung cancer. For the adenocarcinoma group (53 cases), mutation testing for epidermal growth factor receptor (EGFR) was successful in all but one case (with a $21 \mathrm{G}$ needle), i.e. $98.1 \%$ success. Mutation testing for anaplastic lymphoma kinase (ALK) was successful in all 53 cases. Testing for programmed death ligand 1 (PD-L1) was not undertaken at the time of this study.

Benign data showed a higher ratio of subcharacterised benign disease (89.6\% for $19 \mathrm{G}$ compared to $69.8 \% 21 \mathrm{G}$ and $37.9 \%$ for $22 \mathrm{G}$ needle) as shown in Table 5. Sarcoidosis was the most common positive benign diagnosis in all groups ( $85 \% 19 \mathrm{G}$ versus $60 \% 21 \mathrm{G}$ versus $24 \% 22 \mathrm{G})$. The performance in sarcoidosis was higher for $19 \mathrm{G}$ and $21 \mathrm{G}$ than $22 \mathrm{G}$, $95.3 \%$ and $94.1 \%$ versus $87.5 \%$ respectively.

Table 3. Performance data of $19 \mathrm{G}, 21 \mathrm{G}$ and $22 \mathrm{G}$ needles.

\begin{tabular}{lccccc} 
& $\begin{array}{c}\text { Number of false } \\
\text { negative cases } \\
\text { (for malignancy) }\end{array}$ & $\begin{array}{c}\text { Sensitivity } \\
\text { ( } \%)\end{array}$ & $\begin{array}{c}\text { Negative } \\
\text { predictive } \\
\text { Value (\%) }\end{array}$ & $\begin{array}{c}\text { Prevalence } \\
\text { of malignancy } \\
(\%)\end{array}$ & $\begin{array}{c}\text { Accuracy } \\
(\%)\end{array}$ \\
\hline $21 \mathrm{G}$ & 2 & 95.7 & 66.7 & 92.0 & 96.0 \\
\hline $22 \mathrm{G}$ & 3 & 94.7 & 86.2 & 75.0 & 96.0 \\
\hline $\mathrm{p}$ value & 5 & 87.5 & 86.3 & 56.0 & 93.0 \\
\hline
\end{tabular}

Table 4. Subcharacterisation of malignancy 19G, 21G and 22G needles.

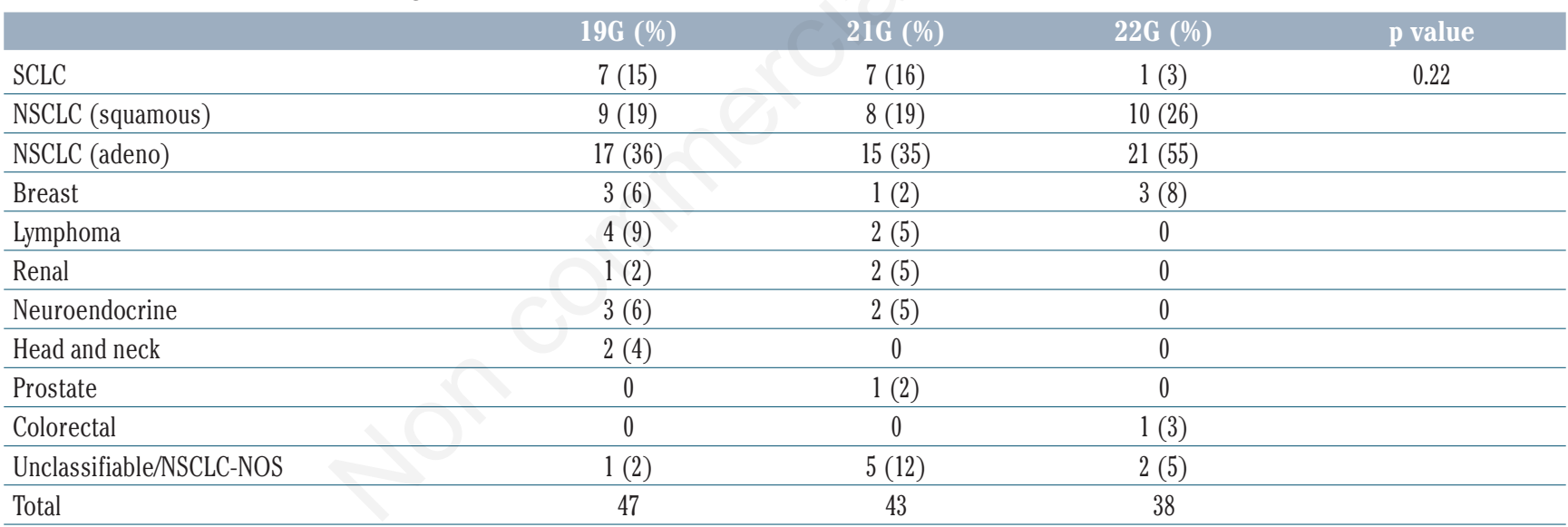

Table 5. Performance data of 19G, 21G and 22G needles in benign disease.

\begin{tabular}{|c|c|c|c|c|}
\hline & $19 G(\%)$ & $21 \mathrm{G}(\%)$ & $22 G(\%)$ & $p$ value \\
\hline Sarcoidosis & $41(85)$ & $32(60)$ & $14(24)$ & 0.07 \\
\hline Tuberculosis & 0 & $1(2)$ & $2(3)$ & \\
\hline $\begin{array}{l}\text { Nodes secondary to interstitial disease } \\
\text { (other than sarcoidosis) }\end{array}$ & $1(2)$ & $2(4)$ & $2(3)$ & \\
\hline Nodes secondary to bronchiectasis/infection & $1(2)$ & 0 & $2(3)$ & \\
\hline Anthracosis & 0 & $2(4)$ & $2(3)$ & \\
\hline Total number of benign nodes subcharacterised & $43(90)$ & $37(70)$ & $22(38)$ & $<0.05$ \\
\hline Unclassifiable/ benign-NOS & $5(10)$ & $16(30)$ & $36(62)$ & \\
\hline Total number of benign cases & 48 & 53 & 58 & 0.37 \\
\hline Total number of undiagnosed sarcoidosis cases & 2 & 2 & 2 & 0.54 \\
\hline Proportion of actual sarcoidosis cases diagnosed & $\begin{array}{c}41 / 43 \\
(95.3 \%)\end{array}$ & $\begin{array}{c}32 / 34 \\
(94.1 \%)\end{array}$ & $\begin{array}{c}14 / 16 \\
(87.5 \%)\end{array}$ & \\
\hline
\end{tabular}




\section{Discussion}

This retrospective observational single centre UK study has demonstrated the $19 \mathrm{G}$ needle appears to have a superior ability to subcharacterise malignant and benign mediastinal lymph nodes compared to $21 \mathrm{G}$ and $22 \mathrm{G}$ needles with a lower rate of NSCLC-NOS. The groups differed slightly in having an older age and smaller lymph node size in the $22 \mathrm{G}$ group; the latter perhaps reflecting case selection of larger nodes for the $19 \mathrm{G}$ by physician choice.

Sensitivity of the $19 \mathrm{G}$ was just slightly higher than $21 \mathrm{G}$ with similar accuracy and both superior to the $22 \mathrm{G}$ needle. Negative predictive value of the $19 \mathrm{G}$ was inferior probably because of the higher prevalence of malignancy in this group again possibly related to the higher mean lymph node size in this group. The higher subcharacterisation rate with the 19G needle allows more tailored and personalised treatment. The success rate for both EGFR and ALK mutation testing in this study were high at $98.1 \%$ and $100 \%$ respectively with only 1 failure with a $21 \mathrm{G}$ needle, in keeping with a previous study from our centre [9]. Historically, EBUS-TBNA has had more limited success [12-14] in the diagnosis of lymphoma but our results with lymphoma (albeit small numbers) are more encouraging perhaps reflecting increased tissue size and integrity with the $19 \mathrm{G}$ needle with less disruption of adjacent tissue. Further studies are required here.

The 19G achieved a higher rate of subcharacterisation for benign mediastinal nodes especially sarcoidosis (with only $10 \%$ without a specific benign diagnosis in the $19 \mathrm{G}$ group). Although this may not translate into treatment as the sarcoidosis was often confined to the mediastinal lymph nodes, it translates into being able to offer patients an explanation for the lymph nodes in a higher number of cases, help clarify length of monitoring and also avoid the need for other more invasive tests.

Finally, we experienced no specific complications with the 19G needle. There was a subjective slight increase in the amount of blood on pathology analysis but not noticeably more bleeding at EBUS-TBNA. No patients required adrenaline, cold saline, transfusion or overnight stay supporting our previous patient satisfaction study for EBUS under conscious sedation and previous initial reports with the $19 \mathrm{G}$ needle alone $[4,6]$.

There are only a handful of published reports on the $19 \mathrm{G}$ needle to date. There are two recent smaller studies that look at the $19 \mathrm{G}$ needle. The first report is of the Polish experience [5], a non-comparative study used the $19 \mathrm{G}$ needle and looked at difference with cell block and smears. It reports 15/22 cases showing malignancy with the remaining 4 showed sarcoidosis and the further 3 cases were diagnosed as reactive - the definition of which is not outlined in the paper. Another small case series of 47 patients again showed safety of the 19G but was not powered to detect superiority over the existing $21 \mathrm{G}$ and $22 \mathrm{G}$ needle data. It showed a diagnostic yield of $89 \%$ for malignancy, $93 \%$ for sarcoidosis, and $83 \%$ for reactive lymph node hyperplasia [15]. A recent study demonstrated that the $19 \mathrm{G}$ core needle is superior to a $21 \mathrm{G} \mathrm{EBUS-}$ TBNA in diagnosis of benign lesions [16].

The strengths of our study are its sample size (largest study so far), comparing to $21 \mathrm{G}$ and $22 \mathrm{G}$ needles too in an unselected real world retrospective setting in an experienced centre. False negatives have been strictly defined with rigorous phenotyping. All procedures were performed by experienced operators and reviewed by experienced lung pathologists in EBUS-TBNA eliminating learning curve factors which are well described in this technique $[17,18]$. All samples were double reported by our lung pathologists.

We acknowledge the limitations of our study which is retrospective, observational and single centre. There was no estimation of optimal sample size for adequate statistical power. Not all needles were used in each patient and our "true positive" malignant results were not validated by mediastinoscopy (standard clinical practice would be not to do this although we acknowledge in very rare cases, false positive EBUSTBNA results can rarely occur because of contamination [19]). Operators were allowed to decide on needle type which may have led to a degree of selection bias and larger nodes being in the 19G group. This may also explain the higher prevalence of malignancy in the 19G group but we acknowledge this may have influenced sensitivity and limited evaluation of the results. Despite these limitations, we feel this real world retrospective study contributes to the field of understanding the potential benefits of the $19 \mathrm{G}$ needle.

\section{Conclusions}

Our study suggests superior subcharacterisation for malignant disease with less NSCLC-NOS cases and better subcharacterisation of benign disease but this requires further study in adequately powered comparative controlled studies with univariate and multivariate analysis. This illustrates the potential benefit of the $19 \mathrm{G}$ needle in achieving a more specific diagnosis as well as avoiding the need for more invasive surgical sampling of the mediastinum to allow more timely personalised treatment with respect to malignant disease and more readily identify treatable benign mediastinal pathology. The time has come for a randomised controlled trial of $19 \mathrm{G}$ versus $21 \mathrm{G}$ and $22 \mathrm{G}$ needles to give further clarity to the latest addition of the EBUS-TBNA armamentarium in particular with respect to performance in lymphoma as well as benign and malignant mediastinal disease.

\section{References}

1. Medford AR, Bennett JA, Free CM, Agrawal S. Mediastinal staging procedures in lung cancer: EBUS, TBNA and mediastinoscopy. Curr Opin Pulm Med 2009;15:334-42.

2. Marchand C, Medford AR. Relationship between endobronchial ultrasound-guided (EBUS)-transbronchial needle aspiration utility and computed tomography staging, node size at EBUS, and positron emission tomography scan node standard uptake values: A retrospective analysis. Thorac Cancer 2017; 8:285-90.

3. Jeyabalan A, Shelley-Fraser G, Medford AR. Impact of needle gauge on characterization of endobronchial ultrasound-guided transbronchial needle aspiration (EBUS-TBNA) histology samples. Respirology 2014;19:735-9.

4. Gnass M, Sola J, Filarecka A, et al. Initial Polish experience of Flexible 19 gauge endobronchial ultrasound-guided transbronchial needle aspiration. Adv Resp Med 2017;85:64-8.

5. Tyan C, Patel P, Czarnecka K, et al. Flexible 19-gauge endobronchial ultrasound-guided transbronchial needle aspiration needle: First experience. Respiration 2017;94:52-7.

6. Jones RC, Medford ARL. The effect of 19G EBUS-TBNA biopsies on characterisation of malignant and benign disease. Eur Respir $\mathrm{J}$ 2017;50:PA382.

7. Jeyabalan A, Medford AR. Endobronchial ultrasound-guided transbronchial needle aspiration: patient satisfaction under light conscious sedation. Respiration 2014;88:244-50.

8. Medford AR, Agrawal S, Free CM, Bennett JA. A performance and theoretical cost analysis of endobronchial ultrasound-guided transbronchial needle aspiration in a UK tertiary respiratory centre. QJM 2009;102:859-64. 
9. Jeyabalan A, Bhatt N, Plummeridge MJ, Medford AR. Adequacy of endobronchial ultrasound-guided transbronchial needle aspiration samples processed as histopathological samples for genetic mutation analysis in lung adenocarcinoma. Mol Clin Oncol 2016;4:119-25.

10. Pillai A, Medford AR. Upcoming endoscopic techniques: endobronchial ultrasound-guided transbronchial needle aspiration. Minerva Pneumol 2011;50:67-82.

11. Steinfort DP, Irving LB. Sarcoidal reaction in regional lymph nodes of patients with non-small cell lung cancer: incidence and implications for minimally invasive staging with endobronchial ultrasound. Lung Cancer 2009;66:305-8.

12. Kennedy MP, Jimenez CA, Bruzzi JF, et al. Endobronchial ultrasound-guided transbronchial needle aspiration in the diagnosis of lymphoma. Thorax 2008;63:360-5.

13. Steinfort DP, Conron M, Tsui A, et al. Endobronchial ultrasoundguided transbronchial needle aspiration for the evaluation of suspected lymphoma. J Thorac Oncol 2010;5:804-9.
14. Karunamurthy A, Cai G, Dacic S, et al. Evaluation of endobronchial ultrasound-guided fine-needle aspirations (EBUS-FNA): Correlation with adequacy and histologic follow-up. Cancer Cytopathol 2014;122:23-32.

15. Jeyabalan A, Medford AR. Endobronchial ultrasound-guided transbronchial needle aspiration: patient satisfaction under light conscious sedation. Respiration 2014;88:244-50.

16. Biswas A, Wynne J, Patel D, Sriram PS. EBUS with 19G Excelon core needle In evaluation of sarcoidosis. Am J Resp Crit Care 2017;A1712.

17. Medford AR. Learning curve for endobronchial ultrasound-guided transbronchial needle aspiration. Chest 2012;141:1643-4.

18. Kemp SV, El Batrawy SH, Harrison RN, et al. Learning curves for endobronchial ultrasound using cusum analysis. Thorax 2010;65:534-8.

19. Sanz-Santos J, Andreo F, Serra P, et al. False positive endobronchial ultrasound-guided real-time transbronchial needle aspiration secondary to bronchial carcinoma in situ at the point of puncture: a case report. J Cardiothor Surg 2012;14;7:74. 